\title{
Acute aortic dissection in a patient with Marfan syndrome during advanced pregnancy
}

\author{
Beti Kostadinovska MD, MSc ${ }^{1}$ | Aleksandar Nikolic MD, $\mathrm{PhD}^{1}$ | Dimche Slaveski MD ${ }^{1} \mid$ \\ Milan Milojevic MD, $\mathrm{PhD}^{1,2}$
}

${ }^{1}$ Department of Cardiac Surgery, Acibadem Sistina Hospital, Skopje, North Macedonia

${ }^{2}$ Department of Cardiothoracic Surgery, Erasmus University Medical Center,

Rotterdam, The Netherlands

\section{Correspondence}

Milan Milojevic, MD, PhD, Department of Cardiothoracic Surgery, Erasmus University Medical Center, Dr. Molewaterplein 40, 3015 GD, P.O. Box 2040, 3000 CA, Rotterdam,

The Netherlands.

Email: m.milojevic@erasmusmc.nl

\begin{abstract}
Pregnant patients with Marfan syndrome (MFS) are at high risk of developing aortic dissection or rupture during the third trimester and early postpartum period. This increased likelihood is the consequence of the hyperdynamic and hypervolemic cardiocirculatory state and/or pregnancy-mediated structural changes of the arterial wall in response to hemodynamic and hormonal changes. In this article, we report on the case of a 26-year-old pregnant woman with MFS in the 30th gestation week, who presented with type A aortic dissection at the emergency department. According to the multidisciplinary team decision, an urgent cesarean section was performed, followed by the Bentall procedure. The patient was discharged on the 10th postoperative day, and her premature child was discharged 6 weeks after birth.

KEYWORDS

aortic dissection, Marfan syndrome, pregnancy
\end{abstract}

\section{1 | INTRODUCTION}

Aortic dissection during pregnancy is unusual and particularly catastrophic, with an estimated maternal prehospital mortality of approximately $50 \%$ and operative mortality of up to $30 \%$. The perioperative fetal mortality rate is even higher and depends on fetal viability. ${ }^{1,2}$ According to results from the Nationwide Inpatient Sample Database, aortic dissection has an average incidence of 0.44 cases per 100000 pregnant women and represents around $0.1 \%$ of aortic dissection cases. ${ }^{3}$ In addition to this, results from the 55 referral centers participating in the International Registry of Acute Aortic Dissection ${ }^{4}$ showed higher rates of aortic dissection in pregnancy of nearly $0.4 \%$ of all cases. Furthermore, the available data suggest that roughly half of the aortic dissections in women younger than 45 years occur during pregnancy, and more than $50 \%$ of these women were affected with the Marfan syndrome (MFS). ${ }^{5}$ Aortic dissection may occur at any time during pregnancy, but it is most frequent in the third trimester (50\%), followed by the postpartum period (20\%) and during labor (15\%). ${ }^{6}$ A review of the current literature shows a substantial difference in the management of acute aortic syndrome during pregnancy (Table 1). Therefore, we present the case of successful surgical treatment of a 26-year-old pregnant woman with an acute type A aortic dissection at 30 weeks of gestation.

\section{2 | CASE REPORT}

A 26-year-old patient (gravida 1, para 0) in the 30th gestation week was referred to the emergency department with a sudden onset of severe anterior chest pain associated with dyspnea. According to information collected from the patient and a family member, she had regular antenatal check-ups and no history of severe illness or accidents. At presentation, the patient had all the clinical features needed to raise a suspicion of MFS. She had a positive family history of aortic disease and sudden death, and her brother underwent genetic testing, which confirmed the diagnosis of MFS.

Emergency echocardiography showed a type A aortic dissection with an entry tear and intimal flap of 14 to $16 \mathrm{~mm}$ above the right coronary artery ostium, resulting in a massive false lumen in the ascending aorta (Figure 1). The diameter of the ascending aorta was $51 \mathrm{~mm}$, left ventricular ejection fraction (LVEF) was preserved, and there was severe aortic valve regurgitation with no sign of 


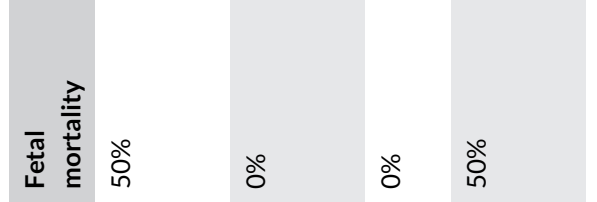

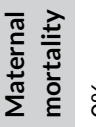

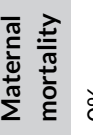

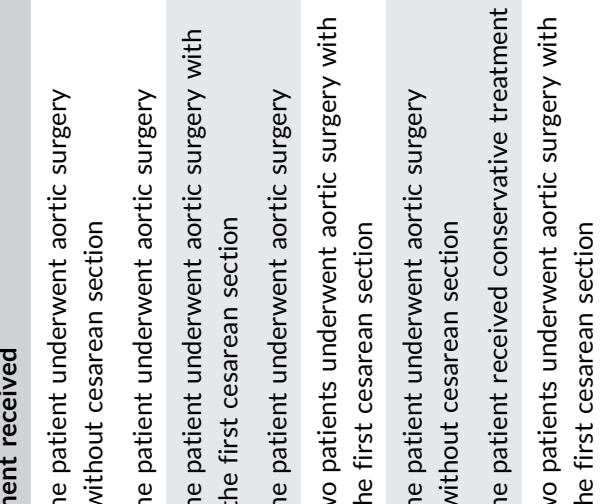

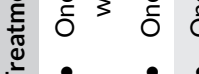

今ั

○े

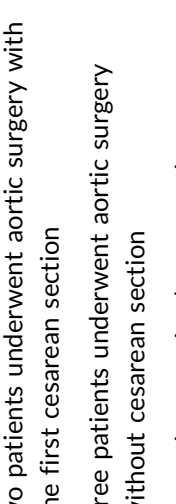

$\frac{\Re}{z}$

๗ั

すั่

品

ळे

১ั
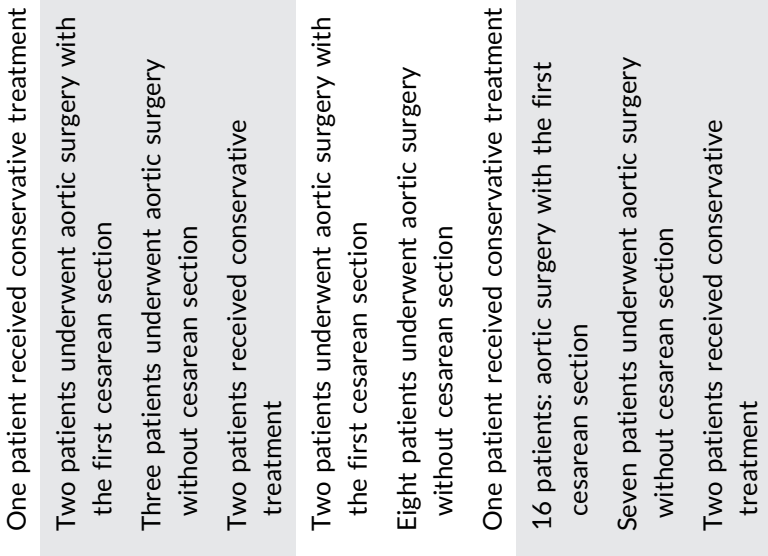

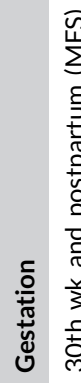
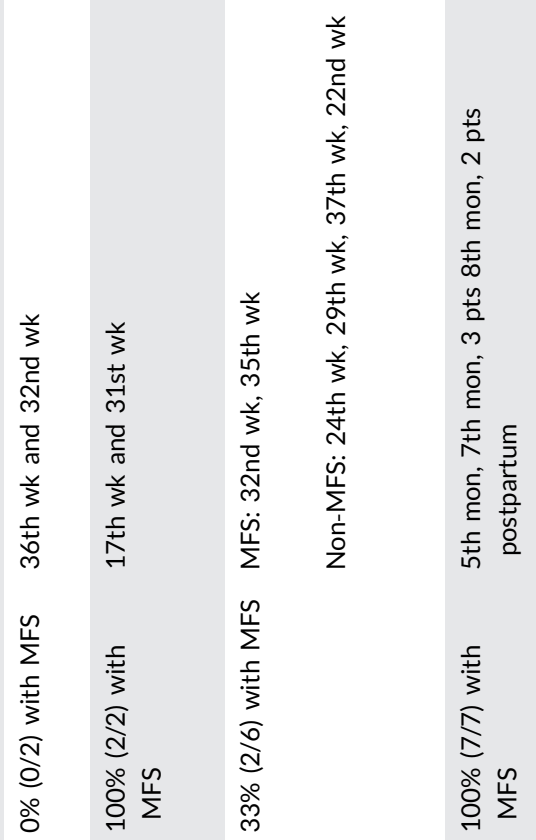

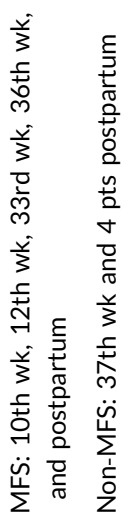

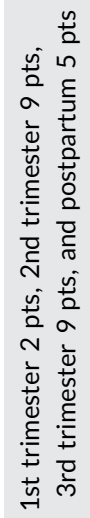

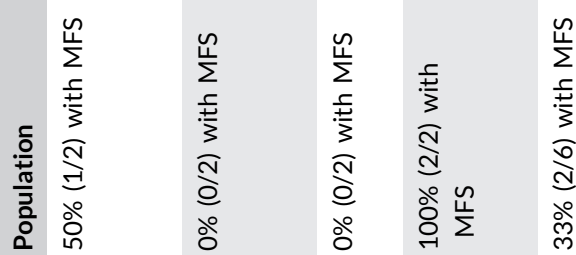
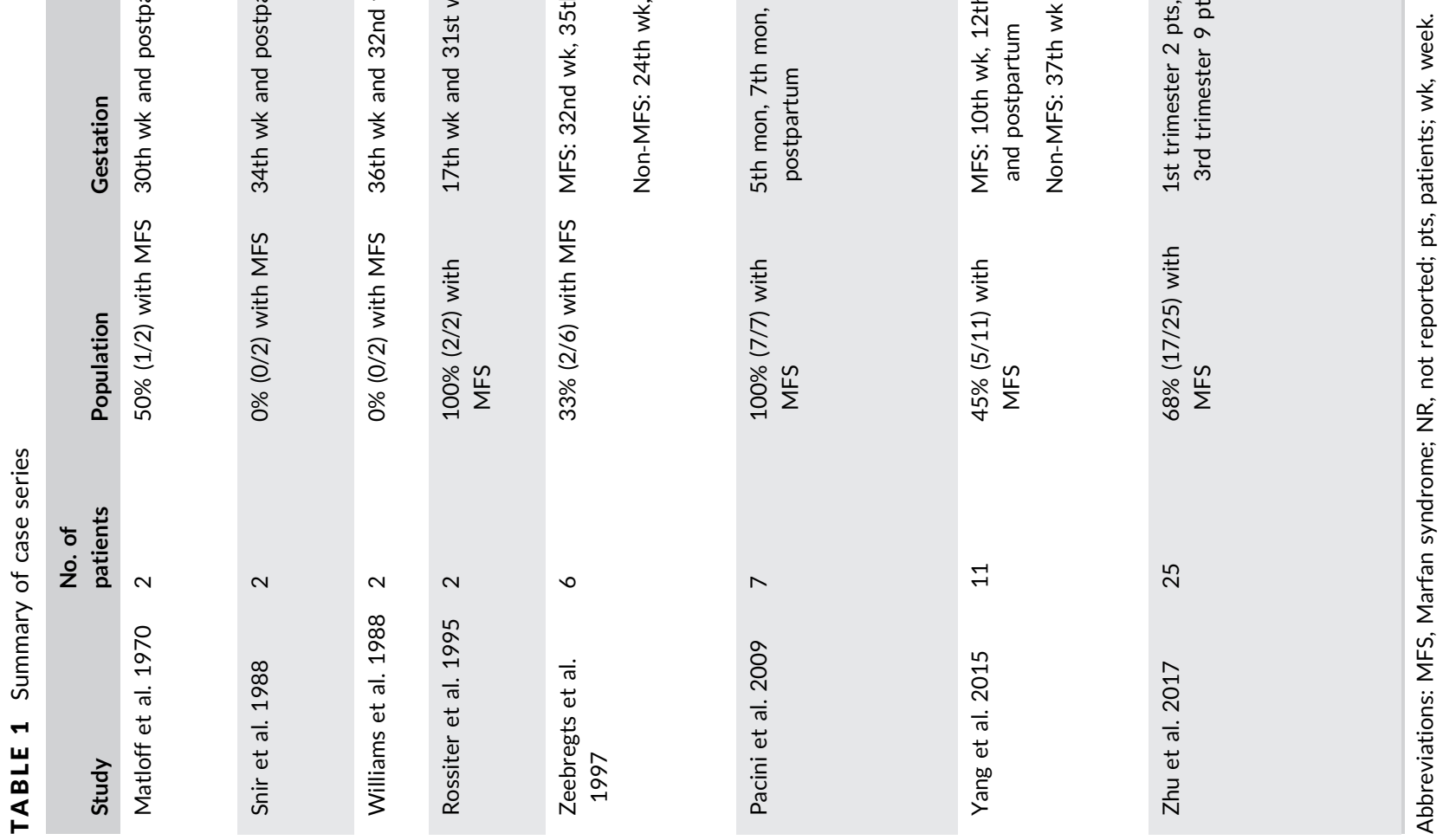


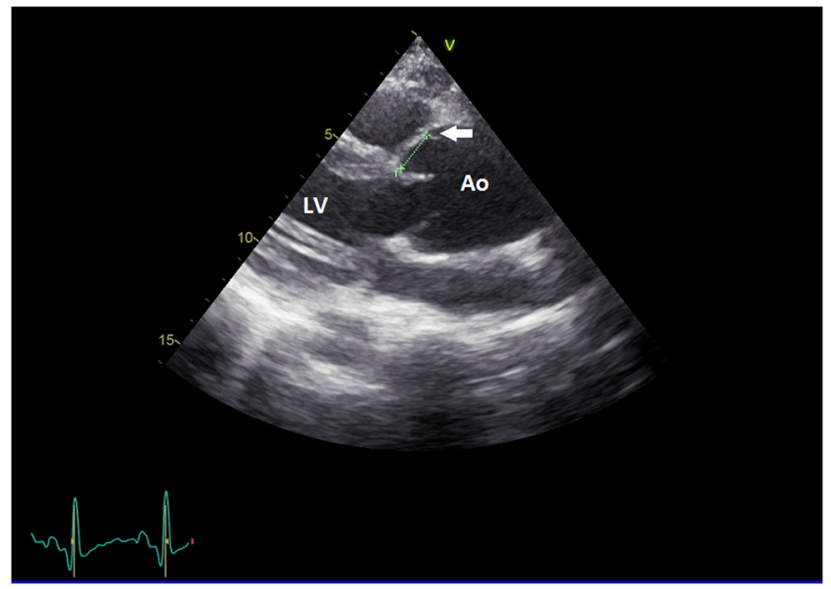

FIGURE 1 Dilated aortic root and intimal flap. Ao, aorta; LV, left ventricle

tamponade (Figure 2). Subsequently, the ultrasound examination showed a viable fetus with a crown-rump length corresponding to the 30th week of gestation, cephalic presentation, and weight around 1700 g. Cardiotocography indicated irregular uterine contractions and a fetal heart rate of $140 \mathrm{bpm}$ with moderate variability. The obstetric team reported that the fetus was stable and viable to adapt to extrauterine life in the neonatal intensive care unit.

A multidisciplinary team including a cardiac surgeon, cardiac anesthesiologist, gynecologist, and pediatrician discussed the various modalities of treatment, and the decision was made to perform a cesarean section followed by cardiac surgery. Immediately after obtaining informed consent for both procedures, the patient was taken to the operating theater. The emergency cesarean section was performed under general anesthesia, using transesophageal echocardiography (TEE) for perioperative monitoring and fluid management (Figure 3). A male infant was born and intubated after 2 minutes of external stimulation due to a failure to initiate spontaneous breathing. He was transferred to the neonatal intensive care ward and placed on mechanical ventilation. After the cesarean section, a modified Bentall procedure was performed using

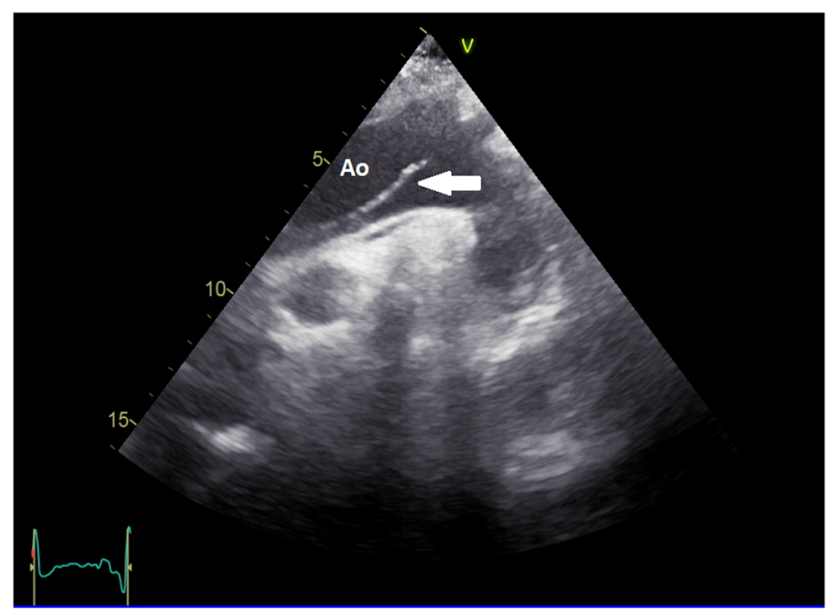

FIGURE 2 Intimal flap ends at the beginning of the truncus brachiocephalicus. Ao, aorta

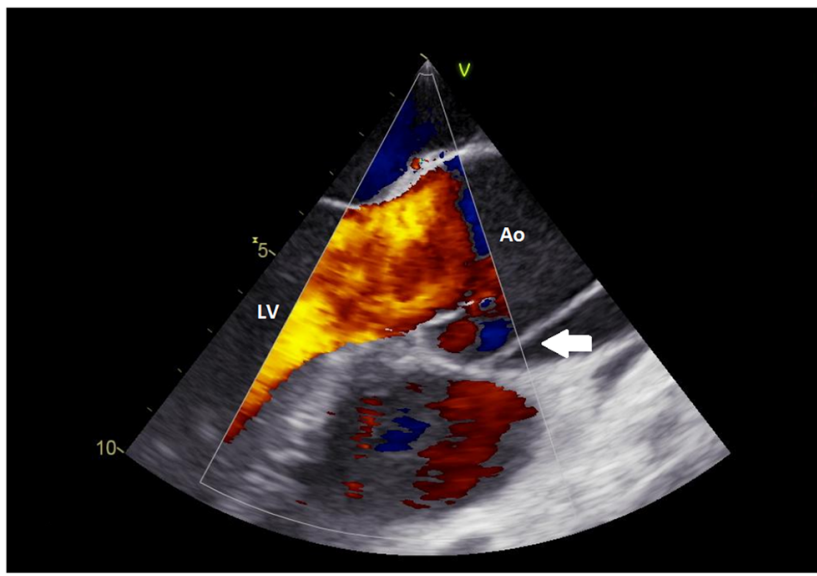

FIGURE 3 Transesophageal echocardiographic mid-esophageal long-axis images of the aortic valve showing aneurysmal dilatation of the aortic root and ascending aorta and intimal dissection flap in the ascending aorta

composite aortic graft with a biological valve prosthesis utilizing Cabrol coronary reimplantation. The decision was made to use a biological valved conduit despite the age of the patient due to her wish to become a parent again. Once rewarming of the patient was completed, weaning from cardiopulmonary bypass (CPB) was accomplished uneventfully. The TEE confirmed a well-functioning aortic prosthesis without any paravalvular leakage and good LVEF. On the sixth postoperative day, a computerized tomography angiogram was performed to confirm the type of aortic dissection (Stanford A, DeBakey II) (Figure 4). No other aneurysmal changes were observed in the descending and abdominal aorta. The patient was discharged from the hospital on the 10th postoperative day in a good general condition. The newborn was discharged home 6 weeks after birth.

\section{3 | DISCUSSION}

The reported rate of aortic dissections in pregnant patients with the MFS is around $5 \%$, with a $50 \%$ chance of a baby being born with the MFS. The risk of aortic dissection is especially high in women with an aortic root diameter greater than $40 \mathrm{~mm}$ before pregnancy. Although

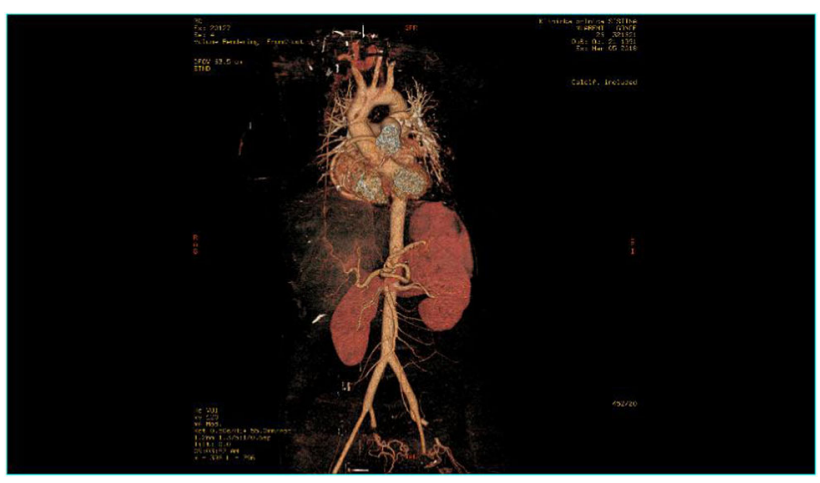

FIGURE 4 Computerized tomography angiography of a patient with DeBakey II aortic dissection 
general risk factors for cardiovascular disease increase a woman's risk for developing aortic dissection during pregnancy, the hyperdynamic and hypervolemic cardiocirculatory state and/or pregnancy-mediated structural changes of the arterial wall in response to hemodynamic stresses and hormonal changes seem to be the main pathophysiological drivers for aortic dissection. Given this risk and that of other cardiovascular complications, American and European Guidelines on the management of cardiovascular disease during pregnancy strongly recommend that pregnant women with MFS should be monitored by echocardiography at 4- to 12-week intervals throughout pregnancy and at 6 months postpartum. The use of beta-blockers to control heart rate and delivery in-hospital with the cardiac surgery department onsite is also highly recommended. Despite all physical features and positive family history, unfortunately, our patient was not diagnosed with MFS, and the above prevention measures were not followed. If a dissection occurs, it is a potentially fatal event for both the mother and the unborn child that requires urgent surgical intervention. Ideally, surgical interventions for aortic dissection should minimize fetal risk without compromising the safety of the mother. Unfortunately, the fetoplacental response to CPB is complex, and fetal loss occurs in nearly $30 \%$ of cases. An appreciation of the risks related to gestational age, including the factors that may influence the survival of the mother and fetus, and close cooperation between a maternal-fetal team and a cardiac surgical team are key components of any strategy used to achieve better outcomes.

The survival rate without severe disabilities among premature babies has markedly increased during the past two decades. In contemporary practice, once the fetus reaches week 25 of gestation, cesarean section, and other active neonatal and obstetric measures are widely used. ${ }^{7}$ Given the low risk of the cesarean section when the mother is in a clinically stable condition, an urgent delivery followed by aortic surgery should be the treatment of choice for all women with aortic dissection in the third trimester of pregnancy (28 weeks). In some circumstances, this is now achievable even earlier, depending on the viability of the fetus. Certainly, this approach provides the best chance of survival for the unborn child and the mother.

\section{4 | CONCLUSION}

Pregnancy in women with MFS is associated with a substantial risk of maternal and fetal complications. Although aortic dissection is uncommon, it is not rare. When it does occur and the fetus is viable, delivery by cesarean section followed by immediate open surgical repair is the current treatment of choice. Timely diagnosis and a multidisciplinary approach are mandatory to achieve satisfactory results in pregnant women affected by aortic dissection.

\section{CONFLICT OF INTERESTS}

The authors declare that there are no conflict of interests.

\section{ORCID}

Milan Milojevic (D) http://orcid.org/0000-0003-1948-9179

\section{REFERENCES}

1. Thalmann $\mathrm{M}$, Sodeck $\mathrm{GH}$, Domanovits $\mathrm{H}$, et al. Acute type $\mathrm{A}$ aortic dissection and pregnancy: a population-based study. Eur J Cardiothorac Surg. 2011;39(6):e159-e163.

2. Elassy SM, Elmidany AA, Elbawab HY. Urgent cardiac surgery during pregnancy: a continuous challenge. Ann Thorac Surg. 2014;97(5): 1624-1629.

3. Sawlani N, Shroff A, Vidovich MI. Aortic dissection and mortality associated with pregnancy in the United States. J Am Coll Cardiol. 2015;65(15):1600-1601.

4. Braverman AC, Harris K, Pyeritz R, et al. Aortic dissection during pregnancy: results from the International Registry of Acute Aortic Dissection (IRAD). J Am Coll Cardiol. 2012;59(13 suppl):E1903.

5. Immer FF, Bansi AG, Immer-Bansi AS, et al. Aortic dissection in pregnancy: analysis of risk factors and outcome. Ann Thorac Surg. 2003; 76(1):309-314.

6. Wanga S, Silversides C, Dore A, de Waard V, Mulder B. Pregnancy and thoracic aortic disease: managing the risks. Can J Cardiol. 2016;32(1): 78-85.

7. Patel RM, Rysavy MA, Bell EF, Tyson JE. Survival of infants born at periviable gestational ages. Clin Perinatol. 2017;44(2):287-303.

How to cite this article: Kostadinovska B, Nikolic A, Slaveski D, Milojevic M. Acute aortic dissection in a patient with Marfan syndrome during advanced pregnancy. J Card Surg. 2019;1-4. https://doi.org/10.1111/jocs.14408 